\title{
ON THE TOPOLOGY AND GEOMETRIC CONSTRUCTION OF ORIENTED MATROIDS AND CONVEX POLYTOPES
}

\author{
JÜRGEN RICHTER AND BERND STURMFELS
}

\begin{abstract}
This paper develops new combinatorial and geometric techniques for studying the topology of the real semialgebraic variety $\mathscr{R}(M)$ of all realizations of an oriented matroid $M$. We focus our attention on point configurations in general position, and as the main result we prove that the realization space of every uniform rank 3 oriented matroid with up to eight points is contractible. For these special classes our theorem implies the isotopy property which states the spaces $\mathscr{R}(M)$ are path-connected.

We further apply our methods to several related problems on convex polytopes and line arrangements. A geometric construction and the isotopy property are obtained for a large class of neighborly polytopes. We improve a result of M. Las Vergnas by constructing a smallest counterexample to a conjecture of G. Ringel, and, finally, we discuss the solution to a problem of R. Cordovil and P. Duchet on the realizability of cyclic matroid polytopes.
\end{abstract}

\section{INTRODUCTION}

This paper deals with the realizability problem of oriented matroids with an emphasis on its topological aspects. This problem is motivated by the fact that several important problems from computational synthetic geometry such as the Steinitz problem for convex polytopes or the embedding of triangulated manifolds can be reduced to oriented matroids [4, 8, 40]. Before plunging into the combinatorial details let us formulate the basic questions in terms of elementary algebra [7].

Given positive integers $n \geq d$, let $\mathbf{R}\left[x_{i j}\right]$ denote the ring of real-valued polynomial functions on a generic $(n-d) \times d$-matrix $X=\left(x_{i j}\right)$. Let $\mathscr{D}_{n, d} \subset$ $\mathbf{R}\left[x_{i j}\right]$ denote the $\left(\begin{array}{l}n \\ d\end{array}\right)-1$ element set of all subdeterminants of $X$ (from order 1 up to order $\min \{n-d, d\})$.

Problem 1.1 (Realizability of oriented matroids). Given any sign assignment $\mathscr{D}_{n, d} \rightarrow\{-, 0,+\}$, does the corresponding system of (sharp) determinant inequalities have a solution in $\mathbf{R}^{(n-d) d}$ ?

Received by the editors July 21,1987 and, in revised form, May 3, 1989.

1980 Mathematics Subject Classification (1985 Revision). Primary 05B35, 52A25; Secondary $14 \mathrm{M} 15,51 \mathrm{H} 99$. 
Once one such solution is found it is very natural to ask for the topological properties of the space of all solutions. This fascinating but very difficult problem had been posed originally for line arrangements by G. Ringel [31], and in a reformulation for projective configurations by R. Pollack [29].

Conjecture 1.2 (Isotopy conjecture [7, 29, 31]). The semialgebraic variety in $\mathbf{R}^{(n-d) d}$ defined by any sign assignment $\mathscr{D}_{n, d} \rightarrow\{-, 0,+\}$ is either empty or path-connected.

Apart from the trivial fact that Conjecture 1.2 holds when $\min \{d, n-d\} \leq$ 2 , only little was known about the topology of such semialgebraic varieties when this paper was first submited in summer 1987. In the meantime the isotopy conjecture has been completely demolished by the counterexamples of White [43], Jaggi and Mani-Levitska [19] and the striking universality theorem of Mnëv [28, 42]. In a short newly added section at the end of the present paper we inform the reader about these recent results and the 1989 state of the art of the subject. Until 1987 Steinitz' classical theorem on the isotopy of convex 3-polytopes was certainly the deepest known result.

Theorem 1.3 (Steinitz [37, §69]). Given two combinatorially equivalent convex 3-polytopes $P_{0}$ and $Q$, there exists a path $\left\{P_{t} \mid 0 \leq t \leq 1\right\}$ of combinatorially equivalent polytopes originating at $P_{0}$ such that $P_{1}$ is equal to either $Q$ or a mirror image of $Q$.

Steinitz' isotopy result can be understood either in the Hausdorff topology on convex bodies in $\mathbf{R}^{3}$ or in the product topology on $\mathbf{R}^{3 n}$ induced from the vertices of the polytopes, both of which agree with the intuitive notion of "closeness" for polytopes of a given type. The generalization of Theorem 1.3 to arbitrary dimensions is essentially equivalent to the Isotopy Conjecture 1.2 by the results of [39], and therefore we restrict our attention to that question.

In [7] J. Bokowski and the second author introduced the concept of solvability sequences as an algorithmic realizability criterion for a large class of oriented matroids, and it is proved in $[7, \S 6]$ that if a uniform oriented matroid $M$ admits such a sequence then its realization space $\mathscr{R}(M)$ is a topological ball. In [40, Chapter 5] solvability sequences are computed (and hence the isotopy conjecture is proved) for all rank 3 uniform oriented matroids with (up to) seven points. However, this criterion is not generally applicable. The first author constructed a realizable rank 3 uniform oriented matroid $M_{9}$ with nine points which does not admit a solvability sequence [5]. (Still, also $\mathscr{R}\left(M_{9}\right)$ is a topological ball.)

A uniform oriented matroid $M$ (or simplicial chirotope in the language of $[4,5,7,8])$ corresponds to a sign assignment $\mathscr{D}_{n, d} \rightarrow\{-,+\}$ in which case the semialgebraic variety $\mathscr{R}(M)$ is an open subset of $\mathbf{R}^{d(n-d)}$. As the main result in the present paper we prove that the realization space $\mathscr{R}(M)$ of a uniform rank 3 oriented matroid $M$ with less than or equal to eight points is contractible (Theorem 4.1). The first author recently extended this result to realizable uniform rank 3 oriented matroids with nine vertices [30]. 
Before giving an overview on the main steps in the proof of this theorem let us first supply some references and basic definitions. Oriented matroids defined by sign patterns as above are also referred to as chirotopes, a term which was coined by A. Dress and A. Dreiding (cf. [13]). Their investigations originated from the study of combinatorial and geometric aspects of chirality in organic chemistry, and it turns out that chirotopes are equivalent (see Lawrence [26]) to oriented matroids which have been axiomatized earlier and independently by Las Vergnas [23], Bland [3], and Folkman and Lawrence [14]. At the same time, also independently, J. Bokowski developed a method based on chirotopes to decide polytopality of a given sphere (Steinitz problem), which was successfully applied in several instances (cf. [4, 8]).

Let $M$ denote a rank $d$ oriented matroid on $E=\{1,2, \ldots, n\}$, and let $\widetilde{R(M)}$ denote the set of real $n \times d$-maticices realizing $M$. The realization space $\mathscr{R}(M)$ is defined as the quotient of the topological space $\widetilde{\mathscr{R}(M)} \subset \mathbf{R}^{\text {nd }}$ by the canonical action of the group $\mathrm{GL}(d, \mathbf{R})$ of nonsingular $d \times d$-matrices.

Deleting a point $e \in E$ in $M$ induces a natural map $\mathscr{R}(M) \rightarrow \mathscr{R}(M \backslash e)$. Each fiber of this map is a convex set, namely it is the residence (cf. Kelly and Moser [20]) of the point $e$ in a realization of $M$. From this it will be derived in $\S 2$ that if the map $\mathscr{R}(M) \rightarrow \mathscr{R}(M \backslash e)$ is surjective (in which case we call $e$ reducible), then the two spaces are homotopy equivalent. Moreover, relating reducibility to the combinatorial concept of sign-invariant pairs in oriented matroids [3, 10, 32], we obtain a generalization of a result of J. P. Roundneff [33, Proposition 5.1] which relates the realizability of rank 3 oriented matroids to the existence of sign-invariant pairs.

In $\S 3$ this approach is generalized to point pairs which are sign-invariant with respect to certain subsets of all cocircuits. A point $e \in E$ will be called isolated if, roughly speaking, it is not contained in any such "locally sign-invariant" pair. With this set-up we prove that every point in a rank 3 uniform oriented matroid $M$ is isolated or reducible. This result, which is due to the first author, yields a powerful and purely combinatorial sufficient condition for reducibility.

In order to show that $\mathscr{R}(M)$ is contractible for an $M$ with eight points, it is now sufficient to find a point $e \in E$ which is not isolated. Since $\mathscr{R}(M)$ is clearly invariant under reorientation, we need only consider representatives from all 135 reorientation classes, and this has been done by exhaustive computer search. Details will be discussed in $\S 4$.

By the results of Folkman and Lawrence [14], the 135 reorientation classes are in one-to-one correspondence with all simple arrangements of eight pseudolines. These arrangements had been classified earlier by Halsey [18], and Goodman and Pollack [16] proved their stretchability, that is, they showed that the spaces $\mathscr{R}(M)$ are nonempty.

The last three sections deal with related geometric problems. In $\S 5$ we apply our methods to the study of neighborly polytopes, and we prove a simple geometric construction and the isotopy property for all neighborly $d$-polytopes with $d+4$ vertices and "sufficiently many" universal edges. Our construction 
applies in particular to all 37 combinatorial types of neighborly 6-polytopes with ten vertices in the classification of Bokowski and Shemer [6].

In [24] M. Las Vergnas gave a counterexample based on oriented matroids to a conjecture of G. Ringel [31] which states that the line slopes in a given type of line arrangement can be prescribed arbitrarily. In $\S 6$ we relate Ringel's problem to our topological investigations, and we give a smallest counterexample with only six lines.

In $\S 7$ we answer a question of $R$. Cordovil and P. Duchet [11] by constructing a nonrealizable cyclic matroid polytope of rank 4 , and we prove that a cyclic matroid polytope is realizable if each of its minors is cyclic.

\section{SOME CONSTRUCTION TECHNIQUES FOR ORIENTED MATROIDS}

A $d$-vector $\Xi$ in $\Lambda_{d} \mathbf{R}^{n}$, i.e., an alternating tensor in the $d$-fold exterior product of $\mathbf{R}^{n}$, is called simple if there are $v_{1}, v_{2}, \ldots, v_{d} \in \mathbf{R}^{n}$ such that $\Xi=v_{1} \wedge v_{2} \wedge \cdots \wedge v_{d}$. The space $\bigwedge_{d} \mathbf{R}^{n} / \mathbf{R}$ of lines through the origin in the vector space $\Lambda_{d} \mathbf{R}^{n}$ is identified as usual with $\left.\left(\begin{array}{l}n \\ d\end{array}\right)-1\right)$-dimensional real projective space. It is well known that the set of simple (projective) $d$-vectors in $\bigwedge_{d} \mathbf{R}^{n} / \mathbf{R}$ is naturally isomorphic to the $(n-d) d$-dimensional Grassmann variety of $d$-dimensional vector subspaces of $\mathbf{R}^{n}$.

Let $M$ be a rank $d$ oriented matroid on $E=\{1,2, \ldots, n\}$, and let $\chi: E^{d} \rightarrow\{-1,0,+1\}$ be the corresponding chirotope [7], that is, the signed bases map of $M$. Write $\mathscr{O}$ for the set of signed circuits and $\mathscr{O}^{*}$ for the set of signed cocircuits of $M$. For every $e \in E$ let $\mathscr{O}_{e}$ denote the set of circuits $C \in \mathscr{O}$ with $e \in C^{+}$, and define similarly $\mathscr{O}_{e}^{*}$.

A simple $d$-vector $\Xi \in \Lambda_{d} \mathbf{R}^{d}$ is a realization of $M$ (or $\chi$ ) provided $\chi=\operatorname{sign} \Xi$. Here the scalar "sign" function is applied componentwise to the Plücker coordinate vector of $\Xi$. The realization space of $M$ (or $\chi)$ is the real semialgebraic variety.

$$
\mathscr{R}(M):=\left\{\Xi \in \bigwedge_{d} \mathbf{R}^{n} / \mathbf{R} \mid \Xi \text { simple and sign } \Xi= \pm \chi\right\} .
$$

This definition agrees with the one given in $\S 1$ where $\mathscr{R}(M)$ was introduced as the topological space of all real vector representations (realizations) $\left\{x_{1}, x_{2}\right.$, $\left.\ldots, x_{n}\right\} \subset \mathbf{R}^{d}$ of $M$ modulo the group action of $\mathrm{GL}(d, \mathbf{R})$. For the Grassmannian of $d$-flats in $\mathbf{R}^{n}$ is parametrized by these equivalence classes of $n \times d$ matrices which can therefore be considered as points in $\bigwedge_{d} \mathbf{R}^{n} / \mathbf{R}$ under the Plücker embedding of the Grassmannian.

The following third equivalent definition of $\mathscr{R}$ is basis-dependent but most practical for actual calculations. Assume w.l.o.g. that $\{1,2, \ldots, d\}$ is a basis of $M$, and let $\left(x_{1}, x_{2}, \ldots, x_{d}\right)$ be any fixed basis of $\mathbf{R}^{d}$. Then we have

$$
\mathscr{R}(M)=\left\{\left(x_{d+1}, \ldots, x_{n}\right) \in \mathbf{R}^{(n-d) d} \mid M=\operatorname{Lin}\left(x_{1}, \ldots, x_{d}, x_{d+1}, \ldots, x_{n}\right)\right\} .
$$

Here "Lin" stands as usual for "oriented matroid of linear dependencies on." 
From this representation of $\mathscr{R}(M)$ it can be seen easily that the realizability problem " $\mathscr{R}(M)=\varnothing$ ?" does lead to the determinantal inequalities in the formulation of Problem 1.1.

As an easy example we have that the realization space of a simplex $(M$ as above with $n=d$ ) is just a point. Observe also that $\mathscr{R}(M)$ is isomorphic to $\mathscr{R}\left(M^{*}\right)$ under the canonical "Hodge star" isomorphism between $\Lambda_{d} \mathbf{R}^{n}$ and $\bigwedge_{n-d} \mathbf{R}^{n}$. As usual, $M^{*}$ denotes the oriented matroid dual to $M$.

A natural approach to the spaces $\mathscr{R}(M)$ is to study possible decompositions related to the combinatorial reduction operations deletion and contraction. Again without loss of generality we may assume that $n>d$ and that $e=n$ is the point to be deleted in $M$. The projection $\mathbf{R}^{(n-d) d} \rightarrow \mathbf{R}^{(n-d-1) d}$ onto the first $(n-d-1) d$ coordinates induces the deletion map $\pi_{n}: \mathscr{R}(M) \rightarrow \mathscr{R}(M \backslash n)$. The deletion map $\pi_{e}$ for any $e \in E$ can also be defined directly in the basis free setting of the spaces $\Lambda_{d} \mathbf{R}^{n}$.

We say that the oriented matroid $M$ is reducible by a point $e \in E$ provided the deletion map $\pi_{e}: \mathscr{R}(M) \rightarrow \mathscr{R}(M \backslash e)$ is surjective. In other words, $M$ is reducible by $e$ if every real realization of the minor $M \backslash e$ extends to a realization to $M$. We have the following useful lemma.

Lemma 2.1. Let $M$ be an oriented matroid and suppose that $M$ is reducible by $e=n \in E$. Then $\mathscr{R}(M)$ is homotopy equivalent to $\mathscr{R}(M \backslash e)$.

Proof. The residence of a point $e \in E$ in a given realization $\Xi \in \mathscr{R}(M)$ of $M$ is the fiber $R_{e, \Xi}:=\pi_{e}^{-1}\left(\pi_{e}(\Xi)\right)$ (compare Kelly and Moser [20]). With the above embeddings of $\mathscr{R}(M)$ and $\mathscr{R}(M \backslash e)$ into $\mathbf{R}^{(n-d) d}$ and $\mathbf{R}^{(n-d-1) d}$ respectively, we can write $\Xi=\left(x_{d+1}, \ldots, x_{n-1}, x_{n}\right)$ with $x_{i} \in \mathbf{R}^{d}$.

With respect to a given realization $\Xi$ there is a linear hyperplane $H(C)$ in $\mathbf{R}^{d}$ corresponding to each signed cocircuit $C \in \mathscr{O}_{e}^{*}$. We write $H_{e, \Xi}(C)$ for the open halfspace in $\mathbf{R}^{d}$ bounded by $H(C)$ and containing $x_{n}$. The residence of $e$ in $\Xi$ can be expressed as the intersection of all these open halfspaces:

$$
R_{e, \Xi}=\bigcap_{C \in \mathscr{O}_{e}^{*}} H_{e, \Xi}(C) .
$$

Using the chirotopy $\chi$ associated with the oriented matroid $M$ we can write equivalently

$$
\begin{aligned}
R_{e, \Xi}=\left\{x \in \mathbf{R}^{d} \mid\right. & \operatorname{sign} \operatorname{det}\left(x_{\lambda_{1}}, \ldots, x_{\lambda_{d-1}}, x\right) \\
& \left.=\chi\left(\lambda_{1}, \ldots, \lambda_{d-1}, n\right) \text { for all } \lambda_{i} \in E\right\} .
\end{aligned}
$$

Thus the residence $R_{e, \Xi}$ of $e$ in $\Xi$ is equal to a convex cone in $\mathbf{R}^{d}$ (or in $\mathbf{R}^{(n-d) d}$ if viewed strictly as a fiber of $\left.\pi_{e}\right)$.

On the other hand, the halfspaces $H_{e, \Xi}(C)$ depend continuously on the parameter vector $\pi_{e}(\Xi)=\left(x_{d+1}, \ldots, x_{n-1}\right) \in \mathscr{R}(M \backslash e)$ and so does their (always nonempty) intersection $R_{e, \Xi}=\pi_{e}^{-1}\left(\pi_{e}(\Xi)\right)$. Consequently, there exists 
a continuous section $\sigma_{e}: \mathscr{R}(M \backslash e) \rightarrow \mathscr{R}(M)$. This means that $\pi_{e} \circ \sigma_{e}$ equals the identity map on $\mathscr{R}(M \backslash e)$.

In order to prove that the deletion map $\pi_{e}$ is a homotopy equivalence between $\mathscr{R}(M)$ and $\mathscr{R}(M \backslash e)$ it remains to show that the map $\sigma_{e} \circ \pi_{e}$ is homotopic to the identity on $\mathscr{R}(M)$. We can interpret $\sigma_{e} \circ \pi_{e}$ geometrically as a continuous "normalization" map which moves (for every realization of the oriented matroid $M$ ) the point corresponding to $e$ into a certain special position within its residence. The fibers $R_{e, \Xi}$ being convex subsets of $\mathbf{R}^{(n-d) d}$, we obtain a homotopy from $\sigma_{e} \circ \pi_{e}$ to $\mathrm{id}_{\mathscr{R}}(M)$ simply by taking the convex combination:

$$
\begin{aligned}
{[0,1] \times \mathscr{R}(M) } & \rightarrow \mathscr{R}(M) \\
\left(t ; x_{d+1}, \ldots, x_{n-1}, x_{n}\right) & \rightarrow\left(x_{d+1}, \ldots, x_{n-1}, t \cdot x_{n}+(1-t)\right. \\
& \left.\cdot \sigma_{e}\left(x_{d+1}, \ldots, x_{n-1}\right)\right) .
\end{aligned}
$$

This completes the proof of Lemma 2.1.

It has been remarked by $R$. Hain that Lemma 2.1 can be obtained directly with standard arguments from the topological theory of fibrations [36]. For, whenever the deletion map $\mathscr{R}(M) \rightarrow \mathscr{R}(M \backslash e)$ is surjective, then it is a fiber bundle with convex (hence contractible) fiber space.

An ordering $\left(e_{1}, e_{2}, \ldots, e_{n}\right)$ of $E$ is called a reduction sequence for $M$ if either $d=n$ or $M$ is reducible by $e_{n}$ and $\left(e_{1}, e_{2}, \ldots, e_{n-1}\right)$ is a reduction sequence for $M \backslash e_{n}$. This notion is very similar to the concept of solvability sequences as introduced in [7]. In fact, a result of J. Richter [5, Proposition 5.2] shows that for rank 3 oriented matroids every solvability sequence induces a reduction sequence. By inductive application of Lemma 2.1 we obtain

Corollary 2.2. If an oriented matroid $M$ admits a reduction sequence, then its realization space $\mathscr{R}(M)$ is contractible.

Let us remark that reducibility is the oriented matroid analogue to the concept of strong coverability for convex polytopes as defined by Altshuler and Shemer [2], and that the statements and proofs of Lemma 2.1 and Corollary 2.2 carry over to realization spaces of convex polytope as well (see [40, Chapter 5] for details). This implies that all convex polytopes obtained from the simplex by strong covering extensions satisfy the isotopy property in the sense of Theorem 1.3. This class includes in particular all 1330 types of 4-polytopes with up to eight vertices [2].

Two distinct elements $e$ and $e^{\prime}$ of a set $E$ are called sign-invariant in an oriented matroid $M$ on $E$ if $e$ and $e^{\prime}$ have always the same sign or always an opposite sign in all signed circuits of $M$ containing them. In the first case the sign-invariant pair $\left\{e, e^{\prime}\right\}$ is called covariant, in the latter contravariant. The covariant pairs of $M$ are contravariant in $M^{*}$ and vice versa. The graph IG $(M)$ on $E$ whose edges are the sign-invariant pairs of $M$ is the inseparability graph of $M[3,10,32]$. A sign-invariant pair of $M$ is sign-invariant in every minor of $M$, and $\operatorname{IG}(M)=\operatorname{IG}\left(M^{*}\right)$. 
Given a realization $X=\left\{x_{1}, \ldots, x_{n}\right\} \subset \mathbf{R}^{d}$ of $M$, then $\{i, j\}$ is signinvariant if and only if $x_{i}$ and $x_{j}$ are separated by either none (contravariant case) or all (covariant case) hyperplanes spanned by elements from $X \backslash\left\{x_{i}, x_{j}\right\}$. In [32] J.-P. Roundneff gave a complete classification of all inseparability graphs of uniform oriented matroids, and he showed that all such graphs can be realized in oriented matroids arising from simplicial polytopes. In [33, Proposition 5.1] he also proved that a rank 3 oriented matroid $M$ is realizable if every minor of $M$ has sign-invariant pairs. Here we obtain the following generalization.

Theorem 2.3. Let $\left\{e, e^{\prime}\right\} \subset E$ be a sign-invariant pair in an oriented matroid $M$ on a finite set $E$. If $M / e^{\prime}$ is reducible by $e$, then $M$ is reducible by $e$.

Before we prove this statement let us note the easy fact that a rank 2 oriented matroid is reducible by each of its points. Thus Roundneff's result is an immediate corollary of Theorem 2.3.

Corollary 2.4 (Roundneff). Let $\left\{e, e^{\prime}\right\} \subset E$ be a sign-invariant pair in a rank 3 oriented matroid $M$ on a finite set $E$. Then $M$ is reducible by $e$, and $M$ is realizable if and only if $M \backslash e$ is realizable.

Proof of Theorem 2.3. Without loss of generality it may be assumed that $E=$ $\{1,2, \ldots, n\}, e^{\prime}=n-1, e=n$, and that $\{n-1, n\}$ is a contravariant pair. We need to show that every linear realization $x_{1}, x_{2}, \ldots, x_{n-1} \in \mathbf{R}^{d}$ of $M \backslash n$ can be extended by a vector $x_{n} \in \mathbf{R}^{d}$ to a realization of $M$.

Let $H \subset \mathbf{R}^{d}$ be the linear hyperplane perpendicular to $x_{n-1}$, and let $p$ denote the orthogonal projection onto $H$. Then $p\left(x_{1}\right), p\left(x_{2}\right), \ldots, p\left(x_{n-2}\right) \in$ $H$ form a linear realization of $(M / n-1) \backslash n$. Since $M / n-1$ is reducible by $n$, there exists a $y \subset H$ such that $\left\{p\left(x_{1}\right), p\left(x_{2}\right), \ldots, p\left(x_{n-2}\right), y\right\}$ is a realization of the rank $d-1$ oriented matroid $M / n-1$ in the hyperplane $H \subset \mathbf{R}^{d}$.

Define $x_{n}:=x_{n-1}+\varepsilon \cdot y$ for some sufficiently small $\varepsilon>0$. By the choice of $y=p\left(x_{n}\right)$, every determinant

$$
\operatorname{det}\left(x_{\lambda_{1}}, \ldots, x_{\lambda_{d-2}}, x_{n-1}, x_{n}\right)=\operatorname{det}\left(p\left(x_{\lambda_{1}}\right), \ldots, p\left(x_{\lambda_{d-2}}\right), x_{n-1}, y\right)
$$

has the correct sign with respect to the chirotope of $M$ for all $\lambda_{i} \leq n-2$. By multilinearity we have on the other hand

$\operatorname{det}\left(x_{\mu_{1}}, \ldots, x_{\mu_{d-1}}, x_{n}\right)=\operatorname{det}\left(x_{\mu_{1}}, \ldots, x_{\mu_{d-1}}, x_{n-1}\right)+\varepsilon \cdot \operatorname{det}\left(x_{\mu_{1}}, \ldots, x_{\mu_{d-1}}, y\right)$

for all $\mu_{i} \leq n-2$. Now the choice of $\varepsilon$ implies

$$
\operatorname{sign} \operatorname{det}\left(x_{\mu_{1}}, \ldots, x_{\mu_{d-1}}, x_{n}\right)=\operatorname{sign} \operatorname{det}\left(x_{\mu_{1}}, \ldots, x_{\mu_{d-1}}, x_{n-1}\right)
$$

whenever both expressions are nonzero. Since $\{n-1, n\}$ was assumed to be contravariant, this shows that all simplices $\left\{x_{\mu_{1}}, \ldots, x_{\mu_{d-1}}, x_{n}\right\}$ have the correct orientation with respect to the chirotope of $M$. This completes the proof of Theorem 2.3. 


\section{ISOLATED POINTS AND REDUCIBILITY}

In this section we introduce the notion of isolated points for uniform oriented matroids, and we prove that if a point $e$ in a rank 3 uniform oriented matroid $M$ is not isolated, then $M$ is reducible by $e$. This yields a purely combinatorial sufficient condition for the reducibility of oriented matroids. In the next section we shall see that this condition is strong enough to guarantee the existence of reduction sequences for all uniform rank 3 oriented matroids with up to eight points. We remark that the techniques developed in this section have also been fundamental for Richter's recent proof of the isotopy property for nine points [30].

Let $M$ be a uniform rank $d$ oriented matroid on $E$, and as before let $\mathscr{O}^{*}$ denote the set of signed cocircuits of $M$. The definition of sign-invariant pairs in the preceding section has the following natural generalization.

Definition 3.1. Let $\Pi \subset \mathscr{O}^{*}$. A pair $\left\{e, e^{\prime}\right\}$ of points of $M$ is sign-invariant relative to $\Pi$ provided $e$ and $e^{\prime}$ have always the same sign or always an opposite sign in all signed cocircuits in $\Pi$ containing them. In the first case $\left\{e, e^{\prime}\right\}$ is contravariant relative to $\Pi$, in the latter case $\left\{e, e^{\prime}\right\}$ is covariant relative to $\Pi$.

Definition 3.2. Let $M, E$, and $\mathscr{O}^{*}$ be as above. We say that a subset $\Pi \subset \mathscr{O}^{*}$ of cocircuits isolates a point $p \in E$ in $M$ provided

(i) $|\Pi|=d$ and

(ii) for all $e \in E$ the pair $\{e, p\}$ is not sign-invariant relative to $\Pi$.

Finally, we call $p \in E$ isolated in $M$ if there is a $\Pi \subset \mathscr{O}^{*}$ which isolates $p$. If $\Pi=\left\{C_{1}, \ldots, C_{d}\right\}$ isolates $p$ in $M$, we also say that $p$ is isolated by the point set $I=C_{1}^{0} \cup \cdots \cup C_{d}^{0}$, that is, the union of all hyperplanes which are involved in the isolation of $p$.

Here $C_{j}^{0}$ denotes the hyperplane complementary to the cocircuit $C_{j}$. Notice that the statement " $p$ is isolated in $M$ by $I \subset E$ " is invariant under reorientations of $M$.

Lemma 3.3. If $p \in E$ is isolated in a uniform rank $d$ oriented matroid $M$ by a point set $I \subset E$, then

$$
|I| \geq\lceil d(d-1) /(d-2)\rceil .
$$

Proof. Let $\Pi=\left\{C_{1}, \ldots, C_{d}\right\}$ be the collection of cocircuits isolating $p$ and with

Abbreviate for $1 \leq i \leq k$

$$
I=\left\{e_{1}, \ldots, e_{k}\right\}=C_{1}^{0} \cup \cdots \cup C_{d}^{0} .
$$

$$
\alpha_{i}:=\left|\left\{C_{j} \in \Pi \mid e_{i} \in C_{j}^{0}\right\}\right| .
$$

The oriented matroid $M$ being uniform, we have $\left|C_{j}^{0}\right|=d-1$ for every cocircuit $C_{j}$, and by counting incidences we get the identity $\sum_{i=1}^{k} \alpha_{i}=\sum_{j=1}^{d}\left|C_{j}^{0}\right|=$ $d(d-1)$. 


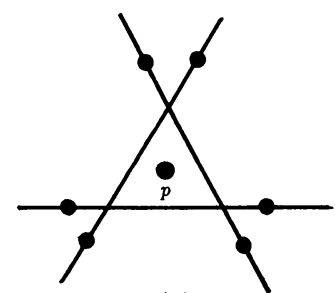

(a)

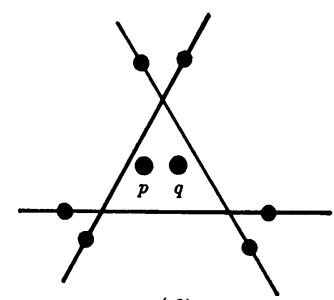

(d)

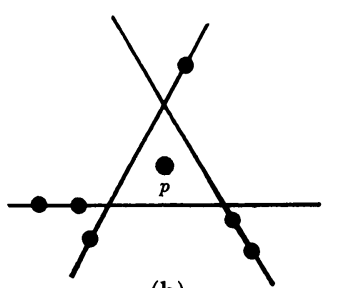

(b)

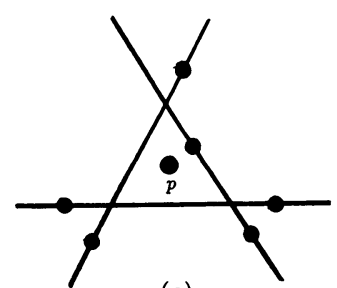

(c)

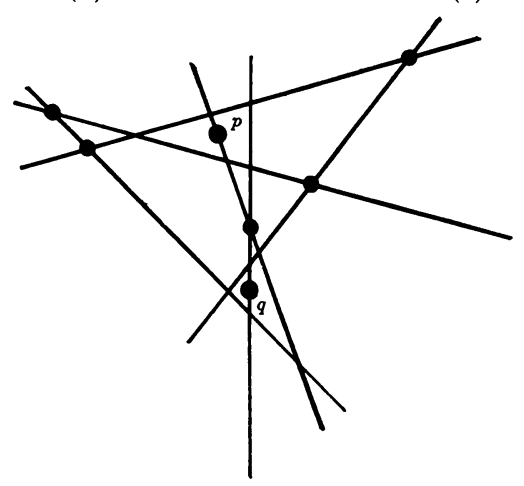

(e)

FIGURE 1. Isolated points in projective diagrams of rank 3 oriented matroids. In (a) and (b) point $p$ is isolated, in (c) and (d) the points $p$ and $q$ are not isolated. In (e) both $p$ and $q$ are isolated.

Every $e_{i}$ is contained in at least two cocircuits from $\Pi$ because otherwise the pair $\left\{p, e_{i}\right\}$ would be sign-invariant relative to $\Pi$. Therefore $\alpha_{i} \leq d-2$. We obtain

and hence

$$
d(d-1)=\sum_{i=1}^{k} \alpha_{i} \leq \sum_{i=1}^{k}(d-2)=k(d-2),
$$

$$
\lceil d(d-1) /(d-2)\rceil \leq k .
$$

A construction due to the first author shows that the bound given in Lemma 3.3 is sharp for every rank $d \geq 3$.

Throughout the remainder of this section we assume that $M$ is a rank 3 uniform oriented matroid. (See Figure 1 for examples of isolated and nonisolated points in the rank 3 case.)

The main result of this section is the following

Theorem 3.4. Let $p$ be a point in a rank 3 uniform oriented matroid $M$. Then $p$ is isolated or $M$ is reducible by $p$.

Combining Lemma 3.3 and Theorem 3.4 we obtain

Corollary 3.5. If $M$ has six or less points, then $M$ is reducible by each of its points. 
In order to prove Theorem 3.4 we need the following combinatorial lemma.

Lemma 3.6. Let $M$ be a uniform rank 3 oriented matroid on $E$ with signed circuits $\mathscr{O}$ and signed cocircuits $\mathscr{O}^{*}$. Let $C \in \mathscr{O}$ with $\underline{C}=\left\{e_{1}, e_{2}, e_{3}, e_{4}\right\} \subset$ $E, p \in C^{0}$, and $C_{1}, C_{2}, C_{3}, C_{4} \in \mathscr{O}^{*}$ such that

(i) $e_{i} \in C_{j}^{+}$for $i, j \in\{1, \ldots, 4\}, i \neq j$, and

(ii) $p \in C_{j}^{+}$for $j \in\{1, \ldots, 4\}$.

Then $C^{+} \neq \varnothing$ and $C^{-} \neq \varnothing$. This means geometrically that the origin is not contained in the interior of the "tetrahedron" $\left\{e_{1}, \ldots, e_{4}\right\}$.

Proof. Assume on the contrary that $C^{-}=\varnothing$ and $C^{+}=\left\{e_{1}, e_{2}, e_{3}, e_{4}\right\}$. Then there exists a positive circuit $X \in \mathscr{O}$ with $X^{+}=\left(C^{+} \cup p\right) \backslash e_{i}$ for some $i \in\{1, \ldots, 4\}$. This can be seen easily by dualizing the restriction of $M$ to $\left\{e_{1}, e_{2}, e_{3}, e_{4}, p\right\}$. This dual is acyclic and of rank 2 , hence its boundary has the form $\left\{p, e_{i}\right\}$. W.l.o.g. we may assume $i=4$, and thus $X^{+}=$ $\left\{p, e_{1}, e_{2}, e_{3}\right\}, X^{-}=\varnothing$.

With (i) we have $C_{4}^{+} \subset\left\{e_{1}, e_{2}, e_{3}\right\}$. Next we apply the orthogonality property (used as an axiom in [3]) of circuits and cocircuits of an oriented matroid, that is, the condition

$$
\left(X^{+} \cap Y^{+}\right) \cup\left(X^{-} \cap Y^{-}\right) \neq \varnothing \Rightarrow\left(X^{+} \cap Y^{-}\right) \cup\left(X^{-} \cap Y^{+}\right) \neq \varnothing
$$

for $X \in \mathscr{O}, Y \in \mathscr{O}^{*}$.

With this, the relation $X^{+} \cap C_{4}^{+}=\left\{e_{1}, e_{2}, e_{3}\right\} \neq \varnothing$ implies

$$
\left(X^{+} \cap C_{4}^{-}\right) \cup\left(X^{-} \cap C_{4}^{+}\right)=X^{+} \cap C_{4}^{-} \neq \varnothing
$$

and hence $p \in C_{4}^{-}$. This contradiction to (ii) completes the proof of Lemma 3.6 .

Now-we are ready for the proof of the main theorem of this section. We use the notations and abbreviations from $\S 2$.

Proof of Theorem 3.4. Suppose $p \in E$ is not isolated in $M$. Then we need to show that the deletion map $\pi_{p}: \mathscr{R}(M) \rightarrow \mathscr{R}(M \backslash p)$ is surjective, i.e., given any realization $\Xi \in \mathscr{R}(M \backslash p)$ of $M \backslash p$, we need to show that the convex cone $\bigcap_{C \in \mathcal{O}_{p}^{*}} H_{p, \Xi}(C)$ is nonempty. Since the open halfspaces $H_{p, \Xi}(C)$ are convex, we can apply Helly's theorem [27, Chapter 1]. It is sufficient to show that the intersection of any four of these halfspaces is nonempty.

Now let $C_{1}, \ldots, C_{4} \in \mathscr{O}_{p}^{*}$ be distinct cocircuits. We abbreviate $\Pi_{i}:=$ $\left\{C_{1}, \ldots, C_{4}\right\} \backslash\left\{C_{i}\right\}$. By assumption, $p$ is not isolated in $M$, and so none of the sets $\Pi_{i}$ isolates $p$. In other words, there exist $e_{1}, \cdots, e_{4} \in E \backslash p$ such that the pair $\left\{p, e_{i}\right\}$ is sign-invariant relative to $\Pi_{i}$ for $i=1,2,3,4$.

Next consider the following sequence of principal extensions $[7,25]$ of $M=$ : $M_{0}$. We set

$$
M_{i}:=M_{i-1}\left[e_{i}^{\sigma_{i}}, p^{+}\right], \quad i \in\{1, \ldots, 4\},
$$


where

$$
\sigma_{i}:= \begin{cases}+ & \text { if }\left\{p, e_{i}\right\} \text { is contravariant relative to } \Pi_{i}, \\ - & \text { if }\left\{p, e_{i}\right\} \text { is covariant relative to } \Pi_{i},\end{cases}
$$

and we denote the new points successively by $n+1, n+2, n+3$, and $n+4$. It follows in particular that the pair $\left\{e_{i}, n+i\right\}$ is sign-invariant in $M_{i}$ and hence also in $M_{i} \backslash p$.

By Corollary 2.4, $M_{i} \backslash p$ is reducible by $n+i$, and we can extend the given realization $\Xi$ of $M \backslash p$ by four new vectors $x_{n+1}, x_{n+2}, x_{n+3}, x_{n+4} \in \mathbf{R}^{3}$ to a realization of $M_{4} \backslash p$. The definition of the oriented matroid $M_{4}$ on $E^{\prime}=$ $\{1,2, \ldots, n+4\}$ via principal extensions implies

(i) $n+i \in C_{j}^{+}$for $i, j \in\{1, \ldots, 4\}$ with $i \neq j$, and

(ii) $p \in C_{j}^{+}$for $j \in\{1, \ldots, 4\}$.

The unique signed circuit $C$ of $M_{4}$ with support $\underline{C}=\{n+1, n+2, n+$ $3, n+4\}$ cannot be positive or negative by Lemma 3.6. After suitable relabelling we may assume that

$$
C^{+}=\{n+1, \ldots, n+m\}, \quad C^{-}=\{n+m+1, \ldots, n+4\}
$$

for some $1 \leq m \leq 4$. Let $x_{n+1}, \ldots, x_{n+4} \in \mathbf{R}^{3}$ be the realization vectors corresponding to $n+1, \ldots, n+4$.

These vectors admit a unique Radon partition which is induced by the signed circuit $\left(C^{+}, C^{-}\right)$. Thus we have a linear dependence of the form

$$
\lambda_{1} x_{n+1}+\cdots+\lambda_{m} x_{n+m}=\lambda_{m+1} x_{n+m+1}+\cdots+\lambda_{4} x_{n+4},
$$

with $\lambda_{i}>0$ for $i \in\{1, \ldots, 4\}$.

Next we pick vectors $a_{i} \in \mathbf{R}^{3}$ for $i=1,2,3,4$ such that the halfspaces $H_{p, \Xi}\left(C_{i}\right)$ are represented as $H_{p, \Xi}\left(C_{i}\right)=\left\{x \in R^{3} \mid\left\langle a_{i}, x\right\rangle>0\right\}$. From the above principal extensions we can read off that $\left\langle a_{j}, x_{i}\right\rangle>0$ for $i \neq j$.

Finally, we define

$$
x_{p}:=\lambda_{1} x_{n+1}+\cdots+\lambda_{m} x_{n+m}=\lambda_{m+1} x_{n+m+1}+\cdots+\lambda_{4} x_{n+4} .
$$

Since the $\lambda_{i}$ are positive, we have $\left\langle a_{j}, x_{p}\right\rangle>0$ for all $j \in\{1, \ldots, 4\}$. Therefore $x_{p}$ is contained in $\bigcap_{i=1}^{4} H_{p, \Xi}\left(C_{i}\right)$, which completes the proof of Theorem 3.4 .

The entire construction is illustrated in Figure 2. In both examples the cocircuits $C_{1}, \ldots, C_{4}$ are represented as darkened lines. The orientations of $p$ with respect to $C_{1}, C_{2}, C_{3}, C_{4}$ are indicated by arrows. The dotted lines represent the Radon partition of the set $\{n+1, \ldots, n+4\}$. Figure $2 b$ demonstrates the necessity of the added points $n+1, \ldots, n+4$ if the cardinality of the set $\left\{e_{1}, \ldots, e_{4}\right\}$ is less than four.

The already very useful criterion given in Theorem 3.4 can still be strengthened. Let $\operatorname{Mut}(M)$ denote the set of mutations of $M$, that is, the set of all bases of $M$ which can be inverted to yield another oriented matroid (see Roundneff 

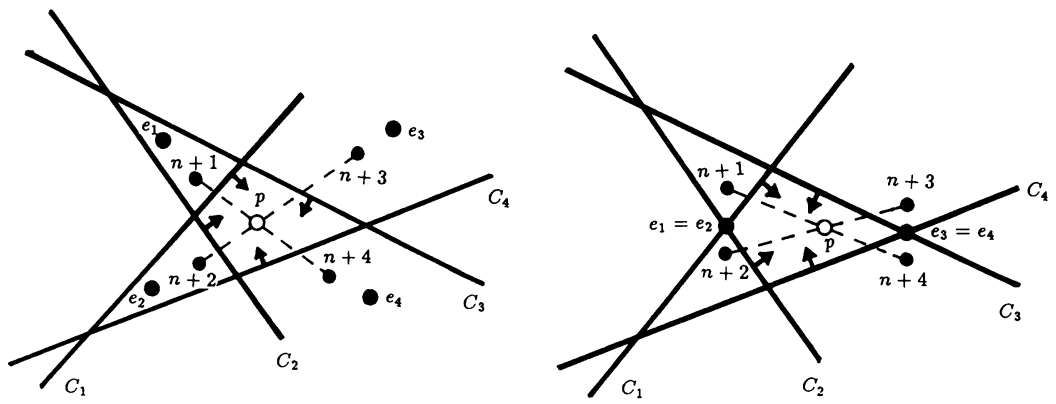

Figure 2. Two examples of the underlying construction of Theorem 3.4

and Sturmfels [34] for a detailed discussion of mutations). It is helpful to note that the mutations of a rank 3 uniform oriented matroid $M$ correspond to the triangles in the arrangement of pseudo-lines associated with $M$. Define

$$
\operatorname{Mut}_{p}(M):=\left\{C \in \mathscr{O}_{p}^{*}(M) \mid C^{0} \cup p \in \operatorname{Mut}(M)\right\} .
$$

Thus $\operatorname{Mut}_{p}(M)$ is a set of cocircuits while $\operatorname{Mut}(M)$ is a set of $d$-tuples of points.

It can be seen that if $p$ is isolated in $M$ by some $\Pi \subset \mathscr{O}^{*}$, then there is also a set $\Pi^{\prime} \subset \operatorname{Mut}_{p}(M)$ which isolates $p$. In fact, this set $\Pi^{\prime}$ can be constructed from $\Pi$ using a straightforward "sweeping-hyperplane-type" algorithm. This implies the following stronger version of Theorem 3.4.

Corollary 3.7. Let $p$ be a point in a rank 3 uniform oriented matroid $M$. Then $p$ is isolated by some $\Pi^{\prime} \subset \operatorname{Mut}_{p}(M)$ or $M$ is reducible by $p$.

\section{PROOF OF THE ISOTOPY PROPERTY FOR SIMPLE ARRANGEMENTS WITH UP TO EIGHT LINES}

Now we are ready to complete the proof of our main result.

Theorem 4.1. Let $M$ be a uniform rank 3 oriented matroid with at most eight points. Then the realization space $\mathscr{R}(M)$ of $M$ is contractible, and hence $M$ satisfies the isotopy property.

While $\S \S 2$ and 3 dealt with the topological and combinatorial ingredients of the proof, it is the aim of this section to outline the computational part which consists of enumerating all oriented matroids in question and detecting reduction sequences. With Corollaries 2.2 and 3.7 we obtain Theorem 4.1 as an immediate consequence of the following.

Lemma 4.2. Let $M$ be a uniform rank 3 oriented matroid on $E=\{1,2, \ldots$, $n\}, n \leq 8$. Then there exists a point $p \in E$ such that $p$ is not isolated by any $\Pi \subset \operatorname{Mut}_{p}(M)$.

First observe that the realization space $\mathscr{R}(M)$ of an oriented matroid $M$ is a reorientation invariant, that is, $\mathscr{R}(M)=\mathscr{R}\left({ }_{A} M\right)$ for all $A \subset E$. If 
$\chi: E^{d} \rightarrow\{-1,+1\}$ is the chirotope of $M$, then the oriented matroid ${ }_{A} M$ is defined by its chirotope $\frac{-}{A} \chi$ as

$$
\begin{aligned}
{ }_{A} \chi: E^{d} & \rightarrow\{-1,+1\} \\
\Delta & \mapsto(-1)^{|\Delta \cap A|} \cdot \chi(\Delta) .
\end{aligned}
$$

Two oriented matroids $M$ and $M^{\prime}$ are equivalent if $M^{\prime}$ is isomorphic to some reorientation ${ }_{A} M$ of $M$. Thus it remains to prove Lemma 4.2 for representatives of all equivalence classes of uniform rank 3 oriented matroids with up to eight points. These equivalence classes are in one-to-one correspondence with all simple arrangements with up to eight pseudo-lines [14, 33].

First observe that if $M$ has at most six points, then Lemma 4.2 (and hence Theorem 4.1) follows directly from Corollary 3.5. The cases with seven and eight points (or lines) have been solved by exhaustive computer search. There are 11 types of simple arrangements with seven lines (see Grünbaum [15, Figure 18.1.1]), and 135 types of simple arrangements with eight lines (see Halsey [18]).

Given a pseudo-line arrangement or oriented matroid $M$, it is a simple task to compute all triangles (= mutations of $M$ ), and this gives us a straightforward subroutine to test the condition in Lemma 4.2. It remained to enumerate all types with seven and eight points. Rather than utilizing the above mentioned lists of Grünbaum and Halsey we used a new enumeration algorithm based on the (local) mutations. This method has the computational advantage that mutations are used twice, namely for testing the condition in Lemma 4.2 and for the enumeration itself.

Given a uniform oriented matroid $M$ and a mutation $\Delta$ of $M$, we write ${ }^{\Delta} M$ for the uniform oriented matroid obtained from $M$ by switching the orientation of the basis $\Delta$ (see $[34, \S 3]$ ). Consider the graph $\mathscr{G}^{n, 3}$ on the set of all uniform rank 3 oriented matroids with $n$ points whose edges are precisely the pairs $\left\{M, \Delta_{M}\right\}$. For $n \leq 8$ all oriented matroids are realizable [16], and hence the graph $\mathscr{G}^{n, 3}$ is connected by [34, Theorem 3.7].

The following recursive algorithm is an adaptation of a standard treetraversing routine applied to the graph $\mathscr{G}^{n, 3}$. Keeping track of the possible mutations gives us full information about neighboring vertices in this graph at each visited node. Finally, to get started, we initialize the main program with the cyclic chirotope (alternating matroid) $\chi \equiv+1$. After termination of the main program, the set $\mathscr{R}$ contains one representative of each uniform oriented matroid equivalence class.

\section{Algorithm 4.3.}

Output: Enumeration of all uniform rank 3 oriented matroids with $n$ points. recursive procedure $\operatorname{SEARCH}(\chi:$ chirotope $)$;

1. $\mathscr{R}:=\mathscr{R} \cup \chi$;

2. $\mathscr{M}:=\operatorname{Mut}(\chi)$;

3. Pick $\Delta \in \mathscr{M} ; \mathscr{M}:=\mathscr{M} \backslash \Delta$; 
4. If ${ }^{\Delta} \chi$ is not equivalent to any element of $\mathscr{R}$

4.1 then SEARCH $\left({ }^{\Delta} \chi\right)$;

5. If $\mathscr{M} \neq \varnothing$

5.1 then goto 3 ;

5.2 else return.

main program;

1. $\mathscr{R}:=\varnothing$;

2. SEARCH $\left(\chi_{n}\right)$, where $\chi_{n}$ denotes the cyclic 3-chirotope with $n$ points;

3. Output $\mathscr{R}$.

Complete lists of all types of arrangements with up to eight lines including coordinates, reduction sequences, $p$-vectors, etc. are available upon request from the first author.

\section{APPLICATIONS TO NEIGHBORLY CONVEX POLYTOPES}

Neighborly polytopes are of considerable importance in the theory of convex polytopes because of their connection with certain extremality properties, the most famous of which is the Upper Bound Theorem due to McMullen [27]. While every neighborly $2 k$-polytope with $2 k+\beta$ vertices is combinatorially equivalent to a cyclic polytope if $\beta \leq 3$, I. Shemer [35, Theorem 6.1] proved that the number $c_{n}(2 k+\beta, 2 k)$ of combinatorial types of neighborly $2 k$-polytopes with $2 k+\beta$ vertices grows superexponentially as $\beta \rightarrow \infty \quad(n \geq 2$ fixed) and as $k \rightarrow \infty$ ( $\beta \geq 4$ fixed). In fact, before the substantial improvements by Goodman and Pollack [17] and Alon [1], Shemer's sewing construction gave the best known lower bound for the number $c(n, d)$ of combinatorial types of all $d$-polytopes with $n$ vertices.

In this section we prove a construction theorem and the isotopy property for a large class of neighborly $2 k$-polytopes with $2 k+4$ vertices. As before, a polytope $P$ is said to satisfy the isotopy property if every polytope $Q$ combinatorially equivalent to $P$ can be connected to either $P$ or a mirror image of $P$ by a path of polytopes of the same type. Besides Steinitz' isotopy theorem and the earlier mentioned results of Altshuler and Shemer [2], it is known that all (even-dimensional) cyclic polytopes satisfy the isotopy property [7, Example 5.1]. Our new construction and isotopy result applies in particular to all neighborly $2 k$-polytopes with $2 k+4$ vertices for $k \leq 3$. The case $k=3$ (37 combinatorial types) has been completely classified in a paper of Bokowski and Shemer [6].

A matroid polytope is an acyclic oriented matroid with only extreme points. All convexity concepts used for matroid polytopes in this section are to be understood in the sense of Las Vergnas [22]. A polytope (or a matroid polytope) is called $m$-neighborly if the convex hull of every $m$-element set of vertices of $P$ is a face of $P$. As is customary in the theory of neighborly polytopes [35, 38], we restrict to the even-dimensional (= odd rank) case. So, by a neighborly polytope we mean a $k$-neighborly $2 k$-polytope $P \subset R^{2 k}$ for some $k$, and similarly for matroid polytopes. 
Given a polytope $P$ and a face $\phi$ of $P$, the quotient $P / \phi$ is a polytope whose face lattice is isomorphic to the upper segment $[\phi, P]$ in the face lattice of $P$. If $P$ is a neighborly $2 k$-polytope with set of vertices $V=$ $\left\{v_{1}, v_{2}, \ldots, v_{n}\right\} \subset R^{2 k}$, an edge $\phi=\operatorname{conv}\left\{v_{i}, v_{j}\right\}$ is called universal [35] if the quotient $P / \phi$ is a neighborly $2(k-1)$-polytope with $n-2$ vertices. Also this definition generalizes to matroid polytopes in the obvious way. In this case quotients are equivalent to contractions. In this section we prove the following result.

Theorem 5.1. (i) Let $M$ be a neighborly matroid polytope of rank $2 k+1$ with $2 k+4$ vertices. If $M$ has at least $2 k-4$ universal edges, then $M$ is realizable and $\mathscr{R}(M)$ is contractible.

(ii) Every neighborly $2 k$-polytope with $2 k+4$ vertices and at least $2 k-4$ universal edges satisfies the isotopy property.

Our proof is based upon the equivalence of universal edges in neighborly polytopes and sign-invariant pairs in the corresponding matroid polytopes which enables us to derive Theorem 5.1 from the results of the previous section. Although this aspect will not be further pursued in the present paper, Theorem 2.3 seems to apply to very many nonneighborly $d$-polytopes with $d+4$ vertices as well. Recall the following characterization of neighborly matroid polytopes.

Lemma 5.2 [11, Lemma 2.8; 38]. (i) A matroid polytope $M$ is m-neighborly if and only if $\left|C^{+}\right| \geq m+1$ for all signed circuits $C$ of $M$.

(ii) $A$ rank $2 k+1$ matroid polytope $M$ is neighborly if and only if $M$ is uniform and $\left|C^{+}\right|=\left|C^{-}\right|$for every signed circuit $C$ of $M$.

Proposition 5.3. Let $M$ be a neighborly matroid polytope. Then $\phi=\left\{v_{i}, v_{j}\right\}$ is a universal edge in the face lattice of $M$ if and only if $\phi$ is a sign-invariant pair of $M$.

Proof. $\phi$ is universal

$\Leftrightarrow M / \phi$ is a neighborly matroid polytope

$\Leftrightarrow\left|X^{+}\right|=\left|X^{-}\right|$for all signed circuits $X$ of $M / \phi$

$\Leftrightarrow\left|Y^{+} \backslash \phi\right|=\left|Y^{-} \backslash \phi\right|$ for all signed circuits $Y$ of $M$ containing $\phi$

$\Leftrightarrow\left|Y^{+} \cap \phi\right|=\left|Y^{-} \cap \phi\right|=1$ for all signed circuits $Y$ of $M$ containing $\phi$

$\Leftrightarrow$ the pair $\phi$ is contravariant in $M$.

Proof of Theorem 5.1. It follows from Theorem 4.1 and Corollary 2.4 and by induction on the number of sign-invariant pairs that a rank 3 oriented matroid $M^{\prime}$ with $n$ points and at least $n-8$ sign-invariant pairs has contractible realization space $\mathscr{R}\left(M^{\prime}\right)$. Apply this to the dual $M^{*}$ of the neighborly matroid polytope $M$ in question. Since $\mathscr{R}(M)=\mathscr{R}\left(M^{*}\right)$, we obtain part (i) of Theorem 5.1 directly from Proposition 5.3.

In order to prove part (ii) we recall the oriented matroid analogue [38, Theorem 4.2] to a beautiful result of Shemer [35, Theorem 2.12] which asserts that every neighborly matroid polytope is rigid, i.e., uniquely determined by its face lattice. So, given a neighborly $2 k$-polytope $P$ with $2 k+4$ vertices and at least 
$2 k-4$ universal edges and associated matroid polytope $M$, the polytopes combinatorially equivalent to $P$ are precisely the realizations of $M$, and in this situation part (i) applies.

In [6] J. Bokowski and I. Shemer gave a complete classification of all 37 (combinatorial types of) neighborly 6-polytopes with ten vertices. Three proofs are given for the nonpolytopality of 14 of the 51 listed neighborly 5 -spheres with ten vertices [6, Theorem 2]. Their third proof implies that the spheres $Q_{38}, Q_{39}, \ldots, Q_{51}$ are not matroid spheres because all 14 contain as a quotient the Brückner 4-sphere with eight vertices which is not a matroid sphere [8]. The 37 matroid spheres $Q_{1}, Q_{2}, \ldots, Q_{37}$ all have at least three universal edges [6, Table 1], and so Theorem 5.1 yields a new polytopality proof and the isotopy property for these 37 spheres.

Corollary 5.4. The unique oriented matroids with face lattices $Q_{1}, Q_{2}, \ldots, Q_{37}$ (see [6]) are realizable, and their realization spaces are contractible. Thus all neighborly 6-polytopes with ten vertices satisfy the isotopy property.

We close this section with an interesting open problem on universal edges in neighborly polytopes. It can be show that "universal edges" in neighborly spheres are a special case of the more general concept of "shrinkable edges" in triangulated spheres, which has been of particular interest recently in connection with the prominent $d$-step conjecture. For a survey on this subject see V. Klee and P. Kleinschmidt [21].

The neighborly 4-polytope $M_{416}^{10}$ with ten vertices (see [8]) is the only known neighborly polytope without universal edges, and it is also the smallest known example of a simplicial polytope without shrinkable edges [21, Theorem 6.4]. It would be interesting to decide whether there exist simplicial $d$-polytopes without shrinkable edges with less than $d+6$ vertices. In the context of this section we suggest the following relaxation.

Problem 5.5. Does there exist a neighborly $2 k$-polytope $P$ with $2 k+4$ vertices such that $P$ has no universal edges?

\section{A SMALl COUNTEREXAMPLE TO A CONJECTURE OF G. RiNGEL}

In [24] M. Las Vergnas disproves a conjecture of G. Ringel by constructing a configuration of points in general position in the plane for which the $x$ coordinates cannot be "prescribed arbitrarily". Originally, this problem was posed in the polar version, that is, it was asked whether the slopes can be prescribed for the lines in a simple arrangement $\mathscr{A}$ in the Euclidean plane [31, p. 102].

To be more precise, let us consider point configurations $\left(P_{1}, \ldots, P_{n}\right)$ in general position in the real plane with $x$-coordinates $x_{1}<\cdots<x_{n}$ such that 
(R) for any sequence $x_{1}^{\prime}<\cdots<x_{n}^{\prime}$ of reals there are $n$ points $P_{1}^{\prime}, \ldots, P_{n}^{\prime}$ in general position in the plane with $x$-coordinates $x_{1}^{\prime}, \ldots, x_{n}^{\prime}$ such that the oriented matroids of affine dependencies on $P_{1}, \ldots, P_{n}$ and $P_{1}^{\prime}, \ldots, P_{n}^{\prime}$ are equal.

Las Vergnas disproves Ringel's conjecture by constructing a configuration with $n=32$ points which does not have property $(R)$. Moreover, he mentions the existence of a similar counterexample with $n=13$, and he asks whether $n=13$ is minimal with this property. This question is interesting in the context of our discussion because if $n=13$ were indeed minimal, then this would imply the isotopy property for uniform rank 3 oriented matroids with up to 13 points.

To see this we observe that prescribing the $x$-coordinates for a realizable rank 3 oriented matroid $\chi$ is equivalent to prescribing coordinates for the rank 2 minor $\Pi / e$ of the point extension $\Pi:=\chi \cup e$ of $\chi$ by a point $e$ "at infinity in the $y$-direction." Once the $x$-coordinates, that is, coordinates for $\Pi / e$, are chosen, it is a linear programming feasibility problem to find $y$-coordinates which extend the $x_{i}$ to a coordinatization of $\Pi$.

When we dualize, then property (R) for $\chi=\Pi \backslash e$ (with $e$ at infinity in the $y$-direction) is equivalent to $e$ being reducible in $\Pi^{*}$. If $e$ is reducible in $\Pi^{*}$ then, by Lemma $2.1, \mathscr{R}\left(\Pi^{*}\right)=\mathscr{R}(\Pi)$ is homotopy equivalent to $\mathscr{R}\left(\Pi^{*} \backslash e\right)=$ $\mathscr{R}(\Pi / e)$. The latter space is clearly contractible since $\Pi / e$ is a rank 2 oriented matroid. This implies

Remark 6.1. Let $n_{0}$ be the largest integer such that $(R)$ is valid for all plane point configurations with up to $n_{0}$ points. Then the isotopy conjecture holds for all rank 3 oriented matroids with up to $n_{0}+1$ points.

However, it turns out that the answer to Las Vergnas' question is negative in the sense that $n_{0}$ is clearly less than 13 .

Theorem 6.2. There is a configuration with $n=6$ points which does not satisfy property (R).

Proof. Consider the affine point configuration $R_{6}^{3}$ in Figure $3 . R_{6}^{3}$ does not satisfy condition $(\mathrm{R})$ because the $x$-coordinates

$$
\begin{aligned}
x_{1}:=-5, & x_{2}:=-3, & x_{3}:=-0.5, \\
x_{4}:=0, & x_{5}:=1, & x_{6}:=6
\end{aligned}
$$

cannot be lifted to a configuration isomorphic to $R_{6}^{3}$.

To see this, we assume on the contrary that there are points

$$
\begin{array}{ccc}
p_{1}=(1,-5, a), & p_{2}=(1,-3, b), & p_{3}=(1,-0.5, c), \\
p_{4}=(1,0, d), & p_{5}=(1,1, e), & p_{6}=(1,6, f),
\end{array}
$$

where $a, \ldots, f \in \mathbf{R}$, and such that

$$
\chi_{R}\left(\lambda_{1}, \lambda_{2}, \lambda_{3}\right)=\operatorname{sign} \operatorname{det}\left(p_{\lambda_{1}}, p_{\lambda_{2}}, p_{\lambda_{3}}\right)
$$




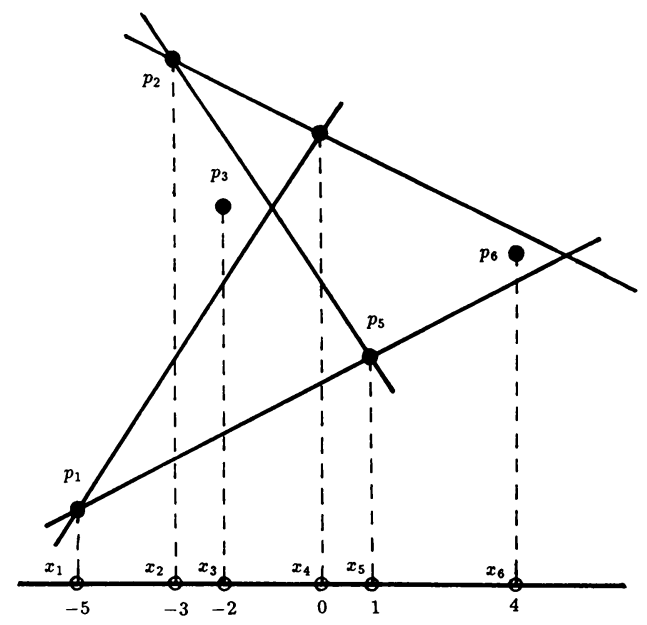

FIGURE 3. A point configuration violating Ringel's property $(\mathbf{R})$

for all $\left(\lambda_{1}, \lambda_{2}, \lambda_{3}\right) \in\{1, \ldots, 6\}^{3}$. Here $\chi_{R}$ denotes the rank 3 oriented matroid of $R_{6}^{3}$. One can read off easily from Figure 3 that

$$
\begin{aligned}
\chi_{R}(1,4,3) & =\chi_{R}(1,5,6)=\chi_{R}(2,3,5) \\
& =\chi_{R}(2,6,4)=\chi_{R}(2,3,4)=+1 .
\end{aligned}
$$

This translates into the inequalities:

$$
\begin{aligned}
& {[143]=-0.5 a \quad+5 c-4.5 d \quad-11 e+6 f>0,} \\
& {[235]=+1.5 b-4 c+2.5 e \quad>0 \text {, }} \\
& {[264]=\quad-6 b+9 d \quad-3 f>0 \text {, }} \\
& {[234]=\quad+0.5 b-3 c+2.5 d \quad>0 \text {. }}
\end{aligned}
$$

Since the linear combination

$$
0=10[143]+[156]+4.4[235]+2[264]+10.8[234]>0
$$

equals zero, we obtain a contradiction. Hence $x_{1}, \ldots, x_{6}$ cannot be lifted to a realization of $\chi_{R}$.

We finish this section by emphasizing the close connection between the above construction and the concept of isolated points. We have seen that for rank 3 oriented matroids one can construct nonreducible points by isolating them. Similar results hold even in higher dimensions.

If we extend $\chi_{R}$ by a point $p_{7}$ at infinity in the $y$-direction and dualize, then we find that $\left(\chi_{R} \cup p_{7}\right)^{*}$ is not reducible by $p_{7}$, and that $p_{7}$ is isolated by the rest of the points. To see this consider the circuits $C_{1}, C_{2}, C_{3}, C_{4}$ of 
$\chi_{R} \cup p_{7}:$

$$
\begin{aligned}
& C_{1}:=(+, 0,-,+, 0,0,+), \\
& C_{2}:=(-, 0,0,0,+,-,+), \\
& C_{3}:=(0,-,+, 0,-, 0,+), \\
& C_{4}:=(0,+, 0,-, 0,+,+) .
\end{aligned}
$$

It follows immediately from Definition 3.2 that $p_{7}$ is isolated by $\left\{C_{1}, \ldots, C_{4}\right\}$ in $\left(\chi_{R} \cup p_{7}\right)^{*}$.

In their paper [12] on the homology of scene analysis H. Crapo and J. Ryan dealt with projective conditions for liftings of linear scenes. A smallest such condition has to be satisfied by six points on a line which can be lifted to a harmonic quadrangle. Our above counterexample is a general position version of that lifting situation.

\section{ON CYCLIC MATROID POLYTOPES AND A PROBLEM OF R. CORDOVIL AND P. DUCHET}

A (matroid) polytope is cyclic if its face lattice is isomorphic to the face lattice of the convex hull of a finite subset of the moment curve $\left\{\left(t, t^{2}, \ldots, t^{d}\right) \in\right.$ $\left.\mathbf{R}^{d} \mid t \in \mathbf{R}\right\}$, and an oriented matroid is alternating if (up to relabelling of the vertices) its chirotope is the constant chirotope $\chi \equiv+1$. (See $[11,15,27,38]$ for terminology and basic properties of cyclic polytopes and their associated oriented matroids.) We have in particular

Remark 7.1 [11, Theorem 3.1; 38, Theorem 4.2]. (i) Every alternating oriented matroid is a cyclic matroid polytope.

(ii) Every cyclic matroid polytope of odd rank is alternating.

The situation is different in the case of even rank. There are, in general, many cyclic matroid polytopes for fixed even rank and number of vertices. The following structural characterization is due to R. Cordovil and P. Duchet.

Theorem 7.2 [11, Theorem 3.2]. A matroid polytope $M$ of even rank is cyclic if and only if $M$ has a covariant pair $\{a, b\}$ such that the contractions $M / a$ and $M / b$ are alternating oriented matroids.

In [11, Remark 5.3] this result is applied to yield geometric constructions for all realizable cyclic matroid polytopes with even rank, that is, all geometrical types of odd-dimensional cyclic polytopes. While Remark 7.1 easily implies that all cyclic matroid polytopes of odd rank are realizable, it remained an open question whether there exist nonrealizable cyclic matroid polytopes of even rank $[11, \S 5]$. It is the aim of this section to settle this question by proving the following

Theorem 7.3. (a) There exists a nonrealizable rank 4 cyclic matroid polytope $M$ with ten vertices.

(b) A rank $2 k$ cyclic matroid polytope $M$ is realizable and $\mathscr{R}(M)$ is contractible if every minor by deletion is cyclic (not necessarily alternating). 
Proof. (a) Let $M_{c}$ denote the rank 4 oriented matroid on $E=\{1,2, \ldots, 9$, $0\}$ of affine dependencies among the row vectors of the following $10 \times 3$-matrix:

$$
\left(\begin{array}{ccc}
2.7 & 2 & 6 \\
3 & 1 & 1 \\
1 & 1 & 0 \\
-1 & 1 & 0 \\
-3 & 1 & 1 \\
-4 & 0 & 1 \\
-1 & -1 & -1 \\
1 & -1 & -1 \\
4 & 0 & 1 \\
3.7 & 0 & -3
\end{array}\right)
$$

The facets of $M_{c}$ are $123,120,134,145,156,167,178,189,190$, $230,340,450,560,670,780,890$, that is, $M_{c}$ is cyclic matroid polytope. The minor $M_{c} \backslash\{1,0\}$ by deletion of the first and the last point is a matroid polytope combinatorially equivalent to a cube: The facets of $M_{c} \backslash\{1,0\}$ are 2345, 2389, 2569, 3478, 4567, 6789. Furthermore, this "matroid cube" has two additional four-point planes, namely 2578 and 3469 . The second author wishes to thank P. Gritzmann for his helpful suggestions on this embedding of the cube into a cyclic polytope.

Now let $\widetilde{M}_{c}$ be the oriented matroid obtained from $M_{c}$ by redefining the orientation of a suitable degenerate "tetrahedron", e.g. 2578 , to be +1 rather than 0 . Since this has no effect on the face lattice, the matroid polytope $\widetilde{M}_{c}$ is cyclic as well. However, $\widetilde{M}_{c}$ is not realizable because $\widetilde{M}_{c} \backslash\{1,0\}$ is a nonrealizable Vamos matroid.

A rigorous nonrealizability proof for $M_{c} \backslash\{0,1\}$ can be given by the following final polynomial $[5,9,40]$ :

$$
\begin{aligned}
& \{29 \mid 5637\}[2437][2987][4937] \\
& -\{23 \mid 5497\}[2967][2987][4937] \\
& +\{27 \mid 5893\}[2967][2934][4937] \\
& +\{93 \mid 6427\}[2437][2987][2957] \\
& -\{98 \mid 6823\}[2437][2957][2934] \\
& +\{37 \mid 4829\}[2967][2957][2934] \\
& =[2956][2437][2987][4937][2937] \\
& +[2354][2967][2987][4937][2937] \\
& +[2758][2967][2934][4937][2937] \\
& + \text { [9364][2437][2987][2957][2937] } \\
& + \text { [9768][2437][2957][2934][2937] }
\end{aligned}
$$

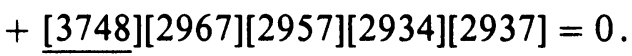


Here the brackets $[a b c d]$ can be interpreted as the oriented volume of the tetrahedron $\{a, b, c, d\}$, and the term $\{a b \mid c d e f\}$ stands for the 3-term Grass-

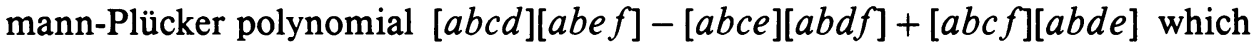
necessarily vanishes in case of realizability. The above expression is a final polynomial, that is, the second identity is impossible because by the construction of the matroid underlying $\widetilde{M}_{c}$ a bracket vanishes if and only if it is underlined.

(b) We use induction on the number of vertices of the cyclic matroid polytope $M$, the result being trivial for simplices. By Theorem 7.2 there exists a covariant pair $\{a, b\}$ of $M$ such that $M / b$ is alternating. Since an alternating matroid is reducible by each of its points [41, Lemma 2], and since $\mathscr{R}(M \backslash a)$ is nonempty and contractible by the induction hypothesis, we can apply Theorem 2.3 to conclude that $\mathscr{R}(M)$ is nonempty and contractible.

We remark that with the same final polynomial one can also produce a nonrealizable uniform cyclic matroid polytope. To this end assign +1 and -1 in such a way to the underlined nonbases that all summands have the same sign.

\section{RECENT RESULTS ON REALIZATION SPACES OF ORIENTED MATROIDS AND POLYTOPES}

This section discusses the recent solutions to the isotopy conjecture for oriented matroids, uniform oriented matroids, polytopes, and simplicial polytopes. These problems have been studied and solved independently by a group of geometers in the west and a group of topologists in the U.S.S.R. We summarize the developments after summer 1987, when the present paper was first submitted for publication.

In July $1987 \mathrm{~N}$. White constructed a rank 3 oriented matroid on $n=42$ points without the isotopy property [43]. Using the Lawrence construction [39] this implied the existence of convex polytopes without the isotopy property. At that time the cases of uniform oriented matroids and simplicial polytopes remained open. During the I.M.A. workshop on "Computational Geometry," Minneapolis, September 1987, L. Lovász indicated that A. M. Vershik's group in Leningrad was studying similar problems, and we inquired about information on their work.

In December 1987 B. Jaggi and P. Mani-Levitska found an example of a uniform oriented matroid without the isotopy property with $n=17$. An easy alternative proof was given in spring 1988 by N. White and B. Sturmfels, and the result was published jointly in [19]. At that time the second author also found a construction method for simplicial polytopes without the isotopy property.

In June $1988 \mathrm{~N}$. White received a short note from A. M. Vershik referring to the articles [28] and [42]. At that time we learned that, far beyond solving the isotopy problem, N. E. Mnëv had proved the following very general and striking universality theorem about realization spaces.

Theorem 8.1 (Mnëv's universality theorem). Let $V$ be any semialgebraic subvariety of $\mathbf{R}^{n}$ which is defined over $\mathbf{Q}$. 
(i) There exists a rank 3 oriented matroid $M$ such that $V$ is stably equivalent to $\mathscr{R}(M)$.

(ii) For some $d \in \mathbf{N}$ there exists a $(d-1)$-sphere $\mathscr{S}$ with $d+4$ vertices such that $V$ is stably equivalent to $\mathscr{R}(\mathscr{S})$.

This theorem can still be strengthened for oriented matroids and polytopes in general position.

Theorem 8.2 (Mnëv's universality theorem, generic version). Let $V$ be any semialgebraic open subvariety of $\mathbf{R}^{n}$ defined by sharp polynomial inequalities over $\mathbf{Q}$.

(i) There exists a uniform rank 3 oriented matroid $\chi$ such that $V$ is stably equivalent to $\mathscr{R}(M)$.

(ii) For some $d \in \mathbf{N}$ there exists a simplicial $(d-1)$-sphere $\mathscr{S}$ with $d+4$ vertices such that $V$ is stable equivalent to $\mathscr{R}(\mathscr{S})$.

We remark that stable equivalence in particular implies homotopy equivalence.

In spite of these striking general results, many interesting questions on realization spaces are still open [42]. We hope that the techniques developed in $\S \S 2-7$ will be useful for answering some of them.

\section{REFERENCES}

1. N. Alon, The number of polytopes, configurations and real matroids, Mathematika 33 (1986), 62-71.

2. A. Altshuler and I. Shemer, Construction theorems for polytopes, Israel J. Math. 47 (1984), 99-110.

3. R. Bland and M. Las Vergnas, Orientability of matroids, J. Combin. Theory Ser. B 24 (1978), 94-123.

4. J. Bokowski, Aspects of computational synthetic geometry, II. Combinatorial complexes and their geometric realization, Presented at the Workshop on Computer-Aided Geometric Reasoning, INRIA, Antibes (France), June 1987.

5. J. Bokowski, J. Richter, and B. Sturmfels, Nonrealizability proofs in computational geometry, Discrete Comput. Geom. 5 (1990), 333-350.

6. J. Bokowski and I. Shemer, Neighborly 6-polytopes with ten vertices, Israel J. Math. 58 (1987), 103-124.

7. J. Bokowski and B. Sturmfels, On the coordinatization of oriented matroids, Discrete Comput. Geom. 1 (1986), 293-306.

8. Polytopal and non-polytopal spheres-An algorithmic approach, Israel J. Math. 57 (1987), 257-271.

9. __ Reell realisierbare orientierte Matroide, Bayreuth. Math. Sch. 21 (1986), 1-13.

10. R. Cordovil and P. Duchet, On sign-invariance graphs of uniform oriented matroids, Discrete Math. 79 (1989/90), 251-257.

11. - Oriented matroids and cyclic polytopes, Combinatorica (to appear).

12. H. Crapo and J. Ryan, Spatial realizations of linear scenes, Structural Topology 13 (1986), 33-68.

13. A. Dress, Chirotops and oriented matroids, Bayreuth. Math. Sch. 21 (1986), 14-68. 
14. J. Folkman and J. Lawrence, Oriented matroids, J. Combin. Theory Ser. B 25 (1978), 199238.

15. B. Grünbaum, Convex polytopes, Interscience, London, 1967.

16. E. Goodman and R. Pollack, Proof of Grünbaum's conjecture on the stretchability of certain arrangements of pseudolines, J. Combin. Theory Ser. A 29 (1980), 385-390.

17. _ Upper bounds for configurations and polytopes in $R^{d}$, Discrete Comput. Geom. 1 (1986), 219-227.

18. E. Halsey, Zonotopal complexes on the $d$-cube, Ph.D. Dissertation, University of Washington, Seattle, 1971.

19. B. Jaggi, P. Mani-Levitska, B. Sturmfels, and N. White, Uniform oriented matroids without the isotopy property, Discrete Comput. Geom. 4 (1989), 97-100.

20. K. M. Kelly and W. O. J. Moser, On the number of ordinary lines determined by $n$ points, Canad. J. Math. 10 (1958), 210-219.

21. V. Klee and P. Kleinschmidt, The d-step conjecture and its relatives, Math. Oper. Res. 12 (1987), 718-755.

22. M. Las Vergnas, Convexity in oriented matroids, J. Combin. Theory Ser. B 29 (1980), 231243.

23. _ _ Matroides orientables, C. R. Acad. Sci. Paris Sér. I Math. 280 (1975), 61-64.

24. __ Order properties of lines in the plane and a conjecture of G. Ringel, J. Combin. Theory Ser. B 41 (1986), 246-249.

25. __ Extensions ponctuelles d'une géométrie combinatoire, Problèmes Combinatoires et Th. des Graphes, Actes Colloq. C.N.R.S., no. 260, Orsay, 1976.

26. J. Lawrence, Oriented matroids and multiply ordered sets, Linear Algebra Appl. 48 (1982), $1-12$.

27. P. McMullen and G. Shephard, Convex polytopes and the upper bound conjecture, London Math. Soc. Lecture Note Series, Vol. 3, Cambridge, 1971.

28. N. E. Mnëv, The universality theorems on the classification problem of configuration varieties and convex polytopes varieties, in Topology and Geometry-Rohlin Seminar (O. Y. Viro, ed.), Lecture Notes in Math., vol. 1346, Springer, Heidelberg, 1988, pp. 527-544.

29. R. Pollack, Problemliste im Tagungsbericht Oberwolfach, Tagung über kombinatorische Geometrie, September 1984.

30. J. Richter, Kombinatorische Realisierbarkeitskriterien für orientierte Matroide, Diplomarbeit, Technische Hochschule Darmstadt, 1988.

31. G. Ringel, Teilungen der Ebene durch Geraden oder topologische Geraden, Math. Z. 64 (1956), 79-102.

32. J.-P. Roundneff, Inseparability graphs of oriented matroids, Combinatorica 9 (1989), 75-84.

33. __ Matroides orientés et arrangements de pseudodroites, Thése der 3em cycle, Université Pierre et Marie Curie, Paris, 1986.

34. J.-P. Roundneff and B. Sturmfels, Simplicial cells in arrangements and mutations of oriented matroids, Geom. Dedicata 27 (1988), 153-170.

35. I. Shemer, Neighborly polytopes, Israel J. Math. 43 (1982), 291-314.

36. E. H. Spanier, Algebraic topology, MacGraw-Hill, New York, 1966.

37. E. Steinitz and H. Rademacher, Vorlesungen über die Theorie der Polyeder, reprint, SpringerVerlag, Berlin, 1976.

38. B. Sturmfels, Neighborly polytopes and oriented matroids, European J. Combin. 9 (1988), 537-546.

39. $\ldots$, On the decidability of diophantine problems in combinatorial geometry, Bull. Amer. Math. Soc. (N.S.) 17 (1987), 121-124.

40. _ Computational synthetic geometry, Ph.D. Dissertation, University of Washington, Seattle, 1987. 
41. __ Cyclic polytopes and d-order curves, Geom. Dedicata 14 (1987), 103-107.

42. A. M. Vershik, Topology of the convex polytopes' manifold, the manifold of projective configurations of a given combinatorial type and representations of lattices, in Topology and Geometry-Rohlin Seminar (O. Y. Viro, ed.), Lecture Notes in Math., vol. 1346, Springer, Heidelberg, 1988, pp. 557-581.

43. N. White, A non-uniform oriented matroid which violates the isotopy property, Discrete Comput. Geom. 4 (1989), 1-2.

Fachbereich Mathematik, Technische Hochschule Darmstadt, Schlossgartenstrasse 7, 6100 DARMSTADT, GERMANY

Department of Mathematics, Cornell University, IthacA, New York 14853-7901 